\section{In-hospital Cardiopulmonary Resuscitation Outcomes}

\section{Janusz Andres ${ }^{1 *}$}

In-hospital cardiopulmonary resuscitation is one the most common medical procedure performed in hospitals. Despite recommended guidelines for reviewing, reporting, and conducting research on in-hospital resuscitation published already in 1997 [1] only recently this subject gained more attention [2]. The overal inhospital resuscitation survival rate with good neurological outcome is low and varies considerably between hospitals. Reported survival to hospital discharge varies from $0 \%$ to $42 \%$ with the most common range being between $15 \%$ and $20 \%$ [3]. There are also differences in survival rates between in-hospital areas like general ward versus emergency department versus intensive care units versus operation room. Definition of in-hospital cardiac arrest or circulatory arrest is very broad, not clearly definied and includes all pulseless cardiac rhythms requiring intervention. The need for chest compressions is the main landmark for inclusion criteria for many in-hospital resuscitation registries. The incidence of treated in-hospital cardiac is rarely reported and values range between 1 and 5 events per 1,000 hospital admissions, or 0.175 events/bed annually [3]. Approximately 200000 US patients annually experience in-hospital cardiac arrest with resuscitation attempt [2]. The most common causes of inhospital cardiac arrest in adults are: cardiac arrhythmia, acute respiratory insufficiency and hypotension mainly because of bleeding [4]. Although a primary arrhythmia is one of the precipitants in nearly half of adult cardiac arrests, only $25 \%$ of in-hospital cardiac arrest victims have ventricular fibrillation or ventricular tachycardia as the initial pulseless rhythm [5]. Pre-resuscitation risk factors associated with increased mortality after in-hospital resuscitation among others are advanced age, pre-existing malignancy, acute stroke, trauma, septicemia, hepatic insufficiency [6]. The crucial question in each case of cardiopulmonary resuscitation attempt is: what is the likehood of survival with a good neurological outcome? There are variables associated with favorable neurological survival after in-hospital resuscitation like younger age, initial cardiac arrest rhythm of ventricular fibrillation or pulseless ventricular tachycardia with a defibrillation time of 2 minutes or less, baseline neurological status without disability, arrest location in a monitored unit, shorter duration of resuscitation, but also the absence of mechanical ventilation, renal insufficiency, hepatic insufficiency, sepsis, malignant disease, and hypotension prior to the arrest. The

\footnotetext{
*Corresponding author: Janusz Andres, Chair and Department of Anaesthesiology and Intensive Therapy, Jagiellonian University Hospital Krakow, Kopernika Str. 17, 31-501 Krakow, Polish Resuscitation Council, Poland, E-mail janusz.andres@uj.edu.pl
}

Received: June 18, 2013 Accepted: June 21, 2013 Published: June 25, 2013 cardiac arrest survival post resuscitation in-hospital score card and normogram for favorable neurological survival has been proposed [7]. The presence of resuscitation system errors like delay in defibrillation and/or vasopressor therapy as well as improper timing in airway management are evident from review of the resuscitation records and are associated with decreased survival from in-hospital cardiac arrest in adults [8]. There is a need for anaesthesia related advanced life support guidelines to be issued in support of the existing resuscitation guidelines [9]. Unanswered questions remain: is cardiac arrest survival the best option to measure and what is the success after the cardiac arrest? Having this in mind we should not forget that no patient whose death is preventable should die in an operation room or hospital - ever [10].

Key points to be considered for in-hospital cardiopulmonary resuscitation issue:

- Monitoring, reporting and reviewing in-hospital resuscitations cases including long term neurological outcome is recommended.

- Patients who witnessed and/or monitored at the time of cardiac arrest demonstrate a higher rate of survival.

- At the bedsite patient specific information should be available to assist in revising resuscitation plans.

- Hospitals should target the training of first responders and code team personel in compliance with resuscitation protocols.

\section{References}

1. Cummins RO, Chamberlain D, Hazinski MF, Nadkarni V, Kloeck W, et al. (1997) Recommended guidelines for reviewing, reporting, and conducting research on in-hospital resuscitation: the in-hospital 'Utstein style'. A statement for healthcare professionals from the American Heart Association, the European Resuscitation Council, the Heart and Stroke Foundation of Canada, the Australian Resuscitation Council, and the Resuscitation Councils of Southern Africa. Resuscitation 34: 151-183.

2. Chan PS, Nallamothu BK (2012) Improving outcomes following in-hospital cardiac arrest: life after death. JAMA 307: 1917-1918.

3. Sandroni C, Nolan J, Cavallaro F, Antonelli M (2007) In-hospital cardiac arrest: incidence, prognosis and possible measures to improve survival. Intensive Care Med 33: 237-245.

4. Goswami S, Brady JE, Jordan DA, Li G (1012) Intraoperative cardiac arrests in adults undergoing noncardiac surgery: incidence, risk factors, and survival outcome. Anesthesiology 117: 1018-1026.

5. Peberdy MA, Kaye W, Ornato JP, Larkin GL, Nadkarni V, et al. (2003) Cardiopulmonary resuscitation of adults in the hospital: a report of 14720 cardiac arrests from the National Registry of Cardiopulmonary Resuscitation. Resuscitation 58: 297-308.

6. Larkin GL, Copes WS, Nathanson BH, Kaye W (2010) Pre-resuscitation factors associated with mortality in 49,130 cases of in-hospital cardiac arrest: a report from the National Registry for Cardiopulmonary Resuscitation. Resuscitation 81: 302-311.

7. Chan PS, Spertus JA, Krumholz HM, Berg RA, Li Y, et al. (2012) A validated 
prediction tool for initial survivors of in-hospital cardiac arrest. Arch Intern Med.172: 947-953

8. Ornato JP, Peberdy MA, Reid RD, Feeser VR, Dhindsa HS, et al. (2012) Impact of resuscitation system errors on survival from in-hospital cardiac arrest. Resuscitation 83: 63-69.
9. Andres J, Hinkelbein J, Böttiger BW (2013) The stepchild of emergency medicine: sudden unexpected cardiac arrest during anaesthesia - do we need anaesthesia-centered Advanced Life Support Guidelines? Eur J Anesthesiol 30: 95-96.

10. Berry WR (2012) Cardiac resuscitation in the operating room: reflections on how we can do better. Can J Anaesth 59: 522-526.

\section{Author Affiliation}

${ }^{1}$ Chair and Department of Anaesthesiology and Intensive Therapy, Jagiellonian University Hospital Krakow, Kopernika Str. 17, 31-501Krakow, Polish Resuscitation Council, Poland

Submit your next manuscript and get advantages of SciTechnol submissions

* 50 Journals

* 21 Day rapid review process

* 1000 Editorial team

* 2 Million readers

* More than 5000 facebook

* Publication immediately after acceptance

* Quality and quick editorial, review processing

Submit your next manuscript at $\bullet$ www.scitechnol.com/submission
This article is published in the special issue, In-hospital Cardiac Arrest has been edited by Dr. Walid Trabelsi, Military hospital of instruction of Tunis, Tunisia 